\title{
ORIGINAL
}

\section{PREVENCIÓN INDICADA DEL CONSUMO PROBLEMÁTICO DE DROGAS EN ADOLESCENTES DE BARCELONA}

\author{
Anna M Guitart (1,2), Montse Bartroli (1,2), Joan R Villalbí (1,2,3), Elvira Guilañá (4), Yolanda \\ Castellano (1,2), Albert Espelt $(1,2,3,5)$ y $M$ Teresa Brugal $(1,2,3)$.
}

(1) Agència de Salut Pública de Barcelona.

(2) Institut d'Investigació Biomèdica Sant Pau, Barcelona

(3) CIBER de Epidemiología y Salud Pública

(4) Institut Genus, Barcelona

(5) Departament de Psicobiologia i Metodologia en Ciencies de la Salut, Universitat Autonoma de Barcelona.

\section{RESUMEN}

Fundamentos: El Servicio de Orientación sobre Drogas (SOD) de Barcelona ofrece un programa de Información y Orientación (PIO) para adolescentes y un Programa de Medidas Alternativas (PMA) a menores sancionados por consumo/tenencia de drogas en la vía pública. E objetivo de este trabajo es describirlos y comparar los perfiles de los usuarios en cada uno de ellos.

Métodos: Estudio transversal descriptivo de 1.010 personas atendidas en ambos programas durante el período 2008-2010, con cribado de patología psiquiátrica y adicción e intervención breve extendida en consumidores sin indicios de patología. Se compararon los perfiles de los usuarios y se calcularon las tasas de uso del PMA por edad.

Resultados: El cannabis motivó el 89,9\% de entradas en el Servicio. Entre los usuarios del PIO el consumo de alto riesgo de cannabis fue del $13,1 \%$ y el consumo de riesgo de alcohol del 11,3\%. Entre los usuarios del PMA los consumos fueron del 8,9 y $4 \%$ respectivamente. El $38 \%$ de los usuarios del PIO y el $6 \%$ de los del PMA se derivaron a atención especializada por presentar criterios de abuso o dependencia a alguna sustancia psicoactiva u otro trastorno psiquiátrico.

Conclusiones: Los adolescentes atendidos en PMA hacen un consumo de cannabis ( $\mathrm{y}$ en muchos casos de alcohol) de riesgo bajo o moderado comparados con los del PIO. Además del valor de la intervención preventiva indicada, los programas facilitan la detección precoz de consumos problemáticos o trastornos mentales incipientes y su derivación.

Palabras clave: Desarrollo de programa. Prevención. Cannabis. Alcohol. Evaluación de programa. Adolescente.

Correspondencia

Joan R Villalbí

Agència de Salut Pública de Barcelona

Pl Lesseps 1

08023 Barcelona

jrvillal@aspb.cat

\section{ABSTRACT}

\section{Indicated Prevention of Problematic Drug Consumption in Adolescents of Barcelona, Spain}

Background: The Drugs Advice Service (SOD in its Catalan acronym) in Barcelona (Catalonia, Spain) provides an Information and Guidance Program (IGP) for teenagers, and an Alternative Measures Program (AMP) targeting minors fined for consumption / possession of illegal drugs in public spaces. This study describes these programs and compares the profiles of their users.

Methods: Cross-sectional descriptive study of 1,010 people discharged from the two SOD programs in 2008-10 after screening for psychiatric disorders and addiction and an extended brief intervention for subjects without pathology. The profiles of the users were compared, and age-specific rates of AMP use were calculated.

Results: Cannabis causes $89.9 \%$ of entries in the SOD. The proportion of IGP users with high risk criteria for cannabis is $13.3 \%$ and with risk for alcohol $11.3 \%$, while in AMP it is $8.9 \%$, and $4 \%$. Criteria for substance dependence or abuse or another psychiatric disorder caused referral of $6 \%$ of AMP users and $38 \%$ of IGP users.

Conclusions: Adolescents in the AMP had a pattern of cannabis use (and often of alcohol) of low or moderate risk, compared to IGP. Beyond the value of the indicated prevention intervention, the programmes facilitate the early detection and referral of problematic substance use and mental disorders in early stages.

Key words: Program development. Prevention. Cannabis. Alcohol. Program evaluation. Adolescent. 


\section{INTRODUCCIÓN}

La adolescencia es un período clave en el desarrollo de la persona, en la que suele tener lugar la experimentación con diversos comportamientos de riesgo, incluyendo el consumo de sustancias adictivas como el tabaco, el alcohol o el cannabis ${ }^{1}$. Al inicio enseñanza secundaria obligatoria (ESO) (en España a los 12 años de edad) la proporción de consumidores de cannabis es relativamente baja, pero asciende notablemente con los años: al final de la ESO el $60 \%$ de estudiantes dice haber probado el cannabis y la proporción que dice haberlo consumido en el último mes supera el 20\% según las encuestas realizadas en Barcelona ${ }^{2}$. Las encuestas ESTUDES sobre muestras de adolescentes de toda España ${ }^{3,4}$ ofrecen datos que confirman este patrón, y además sugieren que desde 1994 hasta 2004 se produjo un incremento en el consumo adolescente, aunque posteriormente se aprecia un ligero descenso. Por otra parte, es habitual el policonsumo, especialmente de cannabis y alcohol.

La invocación a la prevención es habitual, pero ante conductas complejas como el consumo de sustancias con potencial adictivo no todo sirve. En el campo de la prevención del consumo de sustancias adictivas es habitual recurrir al esquema conceptual de tres niveles de prevención: universal, selectiva e indicada ${ }^{5}$. Además, se otorga cada vez más importancia a la prevención ambiental, que modifica el entorno haciendo menos probable el consumo ${ }^{6}$. Más allá de los programas de prevención universal, los programas de prevención indicada se enfocan a las personas que presentan indicios de problemas asociados al consumo de sustancias sin haber desarrollado criterios de dependencia, siendo su finalidad disminuir el consumo de riesgo, reducir la frecuencia de uso y evitar la progresión hacia la dependencia ${ }^{7}$. Entre dichos programas, destacan los que se basan en intervenciones breves, en sus diferentes modalidades, y los que comportan interven- ciones sobre la familia ${ }^{8,9}$. Las intervenciones breves han demostrado su efectividad y eficacia en diversos estudios, especialmente para reducir el consumo de alcohol de riesgo $^{10,11}$. La aceptación de este tipo de intervenciones en adolescentes y jóvenes en riesgo ha permitido su adaptación en diversos contextos, como las salas de urgencias ${ }^{12,13}$, atención primaria sanitaria ${ }^{14}$, justicia juvenil $^{15} \mathrm{o}$ en entornos escolares ${ }^{16}$.

Con una perspectiva orientada a la prevención indicada, los servicios de salud pública de la ciudad de Barcelona pusieron en marcha en el año 2000 el Servicio de Orientación sobre Drogas (SOD) creado para abordar la problemática planteada por el consumo de alcohol y otras drogas de los adolescentes. Su objetivo fue responder a las demandas de información y orientación de los adolescentes y jóvenes y sus familias, habitualmente centradas en problemas de consumo de cannabis sin una dependencia establecida ${ }^{17}$. En la práctica, cabalgaba entre la prevención selectiva y la indicada, y el tratamiento precoz (con algún solapamiento con otros programas), hasta los 21 años de edad. Por ello se reformuló buscando que encajara mejor con los otros servicios existentes en la ciudad. El nuevo enfoque trabaja con un modelo de intervención breve extendida basada en estrategias motivacionales $^{18}$, orientada a prevenir el consumo problemático de sustancias adictivas en adolescentes con experiencia de consumo ${ }^{19}$, complementando así con una oferta de prevención indicada los programas de prevención universal ofrecidos en la escuela ${ }^{3}$.

Buscando cómo contactar y atender mejor a adolescentes en riesgo en el momento apropiado, se inició una experiencia con los servicios de mediación de justicia juvenil y en 2008 se puso en marcha un convenio de colaboración con los cuerpos de seguridad (Mossos d'Esquadra y Guardia Urbana) que se diseñó fundamentándose en estudios previos que indicaban que el $61,5 \%$ de los consumidores habituales de cannabis 
se habían iniciado en grupo en la vía públi$\mathrm{ca}^{20}$ y que tanto el cambio ambiental para hacer menos fácil el consumo, como el control de la oferta son medidas preventivas probadas $^{5,21}$. Este convenio dio lugar en el SOD al nuevo Programa de Medidas Alternativas (PMA), que facilita a los menores de edad denunciados por consumo o tenencia de sustancias psicoactivas ilegales en la vía pública la posibilidad, por una sola vez, de acceder a una atención psicoeducativa como medida alternativa a la sanción administrativa.

En la actualidad coexisten en el SOD dos programas basados en una intervención breve extendida. El nuevo Programa de Medidas Alternativas (PMA), al que se acumula el anterior Programa de Medidas Alternativas a las Judiciales (PMA-J) que acoge pocos casos, y el inicial Programa de Información y Orientación (PIO) para adolescentes, jóvenes y sus familias, que se mantiene con una mayor protocolización de sus actividades.

El objetivo de este estudio es describir los dos programas del SOD y comparar los perfiles de sus usuarios, así como conocer si el PMA permite captar mejor a consumidores que se encuentran en etapas previas al desarrollo de dependencia u otra patología psiquiátrica.

\section{SUJETOS Y MÉTODOS}

\section{Descripción de los programas y las} intervenciones. El equipo asistencial está formado por psicólogos clínicos con experiencia en atención a las drogodependencias. Ambos programas se basan en una intervención breve extendida en sujetos con consumo y sin indicios de patología. El objetivo de la intervención es la motivación para el cambio en relación al consumo de sustancias y la información y asesoramiento al adolescente y su familia, abriendo un espacio de reflexión sobre el consumo, su función y sus posibles efectos en los dife- rentes ámbitos de la vida. El número de sesiones es de 4 a 6 , y se hace una primera visita de acogida en presencia de los padres o tutores legales, durante la que se realiza una entrevista clínico-epidemiológica con el cribado del consumo de riesgo de alcohol mediante el cuestionario Alcohol Use Disorders Identification Test reducido (AUDIT-C) ${ }^{22}$, el cribado de consumo de riesgo de cannabis con la escala Cannabis Abuse Screening Test (CAST) ${ }^{23}$ y el cribado de posibles psicopatologías con el cuestionario Problem oriented screening instrument for teenagers (POSIT) ${ }^{24}$-en dos áreas funcionales: el estado de salud mental y las conductas agresivas y delincuentes-, haciendo especial hincapié en las conductas de riesgo y en la detección de psicosis incipiente. En el supuesto de que se detecte alguna patología se deriva al sujeto a los recursos asistenciales adecuados.

Las sesiones de seguimiento se realizan individualmente con el menor. Se sigue el modelo de estadíos del cambio de Prochaska y DiClemente ${ }^{25}$, orientando a partir de la reflexión personal cambios de estilos de vida y actitudes más favorables y saludables. La intervención clínica puede ampliarse en aquellos casos que sea necesaria por su urgencia, por situaciones de riesgo o por la derivación a otro recurso. La última sesión es de conclusión y de valoración del proceso. Los padres o tutores legales han de asistir al menos a la primera y/o última visita.

Sujetos y criterios de inclusión. Estudio transversal de las personas atendidas y dadas de alta en los programas del SOD a lo largo de 30 meses $(n=1.010)$, de julio de 2008 a diciembre de 2010. Los criterios de inclusión para el subprograma de PMA fueron: haber sido denunciados por tenencia o consumo de drogas ilegales en el espacio público, tener de 14 a 17 años en el momento de la denuncia, residir en Barcelona ciudad y ser la primera vez que se acogían al programa (no se admitían reincidentes). En este período hubo también 9 casos deriva- 
dos por los servicios de justicia juvenil al PMA-J que, por los escasos efectivos y su similitud conceptual, se trataron conjuntamente con los del PMA. Los adolescentes atendidos en el PIO podían acceder por iniciativa propia o de la familia, o por derivación desde otros recursos sanitarios, sociales y educativos. Los únicos criterios de inclusión en el PIO fueron ser menor de 22 años y residir en Barcelona. No se incluyó en este estudio la actividad de acogida y asistencia a familias que no culminara en atención presencial a un adolescente o joven.

Variables. Las variables estudiadas se extrajeron de la documentación del SOD. Además del tipo de programa, incluyeron variables sociodemográficas (sexo, edad, nacionalidad, estructura familiar, estudios y actividades extraescolares organizadas), variables clínicas (antecedentes de tratamiento psiquiátrico o psicológico y variables relacionadas con el consumo previo al programa (sustancia que suscita el contacto, consumo de otras sustancias, patrón de consumo de cannabis y de alcohol, consumo de cannabis de riesgo (CAST $)^{23}$ y consumo de alcohol de riesgo (AUDIT-C) ${ }^{22}$. Con el AUDIT-C, al aplicarse en población adolescente con mayor susceptibilidad, se opta por un valor superior o igual a 4 como valor de corte Finalmente se incluyeron variables que reflejaban el producto inmediato del programa a corto plazo, incluyendo la derivación a otros centros especializados en adicciones o salud mental por presentar indicios de patología y la valoración del cumplimiento efectivo del programa.

Análisis estadístico. Para el estudio se analizaron las variables comparando los perfiles de los usuarios de los dos programas (PIO y PMA) mediante la prueba $\chi 2$. Se calcularon las tasas de uso del PMA específicas por edad y sus IC $95 \%$, utilizando como denominador la población de Barcelona del año 2010 para cada edad ${ }^{26}$. Para los análisis estadísticos se utilizó el paquete estadístico SPSS18.

\section{RESULTADOS}

A lo largo de los 30 meses incluidos en eset estudio, el servicio atendió 1.010 casos (34 nuevos usuarios al mes), 907 (89,8\%) casos en el PMA, de los que 9 procedieron del PMA-J y 103 (10,2\%) del PIO. Los usuarios del PMA fueron básicamente menores (un $4 \%$ cumplieron 18 años en el periodo que fue de su derivación hasta el inicio el programa), mientras que en el PIO el 37,3\% fueron mayores de 18 años. Las características de las personas atendidas en cada subprograma se presentan en la tabla 1. De los 1.010 usuarios del SOD, 858 (85\%) fueron varones, $793(78,5 \%)$ estaban cursando estudios y $935(92,6 \%)$ tenían nacionalidad española 405 (40\%) mencionaron haber recibido atención previa psicológica y/o psiquiátrica, y 160 (15\%?) había tenido contacto con servicios sociales o equipos de protección a la infancia.

Comparando los participantes de los dos programas se apreciaron diferencias estadísticamente significativas en diversas variables. La sustancia que motivó un mayor número de entradas al PMA fue el cannabis en $900(99,2 \%)$ sujetos. Otras variables con diferencias estadísticamente respecto al PIO fueron pertenecer a una familia nuclear (formada por padre, madre e hijo/s) y realizar algún tipo de actividad reglada en el tiempo de ocio. No se observaron diferencias estadísticamente significativas entre programas en lo que respecta al sexo, nacionalidad, atención psiquiátrica y/o psicológica previa, ni en intervención de los servicios sociales o de equipos de protección a la infancia.

El consumo de cannabis o de alcohol con criterios de riesgo fue más frecuente en el PIO. Se consideró consumo de cannabis de alto riesgo (CAST > 4) en $10(13,1 \%)$ usuarios, y con el AUDIT-C se valoró que 8 $(11,3 \%)$ tenían criterios de consumo de alcohol de riesgo. En el PMA fueron $71(8,9 \%)$ y $35(4 \%)$ respectivamente. Por 


\section{Tabla 1}

Características de las personas atendidas en el Servicio de Orientación sobre Drogas según programa. Barcelona, 2008-10

\begin{tabular}{|c|c|c|c|}
\hline & $\begin{array}{c}\text { Programa de } \\
\text { Medidas Alternati- } \\
\text { vas (PMA) } \\
\mathrm{N}=907\end{array}$ & $\begin{array}{c}\text { Programa de } \\
\text { Información y } \\
\text { Orientación (PIO) } \\
\mathrm{N}=103\end{array}$ & $\mathrm{p}$ \\
\hline Varones & $778(85,8 \%)$ & $80(77,7 \%)$ & $<0,03$ \\
\hline Nacionalidad española & $772(85,8 \%)$ & $86(83,7 \%)$ & NS \\
\hline Familia monoparental & $232(26,0 \%)$ & $29(29,9 \%)$ & \multirow{2}{*}{$<0,05$} \\
\hline Familia nuclear & $536(60,1 \%)$ & $47(48,5 \%)$ & \\
\hline Cursando estudios & $724(80,2 \%)$ & $69(66,9 \%)$ & $<0,05$ \\
\hline Participación en actividades extraescolares organizadas & $398(43,9 \%)$ & $27(26,2 \%)$ & $<0,0001$ \\
\hline Tratamiento psicológico y/o psiquiátrico previo & $373(41,8 \%)$ & $32(36,8 \%)$ & NS \\
\hline $\begin{array}{l}\text { Intervención previa de servicios sociales o equipos de protección } \\
\text { a la infancia }\end{array}$ & $148(16,8 \%)$ & $12(15,6 \%)$ & NS \\
\hline Cannabis como sustancia que motiva el inicio de programa & $900(99,2 \%)$ & $85(92,4 \%)$ & $<0,0001$ \\
\hline Consumo de cannabis de alto riesgo (CAST $>3$ ) & $71(8,9 \%)$ & $10(13,1 \%)$ & \multirow[b]{2}{*}{$<0,0001$} \\
\hline $\begin{array}{l}\text { Consumo de cannabis de bajo riesgo o riesgo moderado (2-3 } \\
\text { puntos en CAST) }\end{array}$ & $182(22,7 \%)$ & $12(13,2 \%)$ & \\
\hline Consumo de alcohol de riesgo (AUDIT-C $>4$ ) & $35(4,0 \%)$ & $8(11,3 \%)$ & $<0,01$ \\
\hline
\end{tabular}

\section{Figura 1}

Sustancias notificadas como consumidas en los 30 días anteriores a la primera visita por las personas atendidas en el Servicio de Orientación sobre Drogas según programa de atención. Barcelona, 2008-10

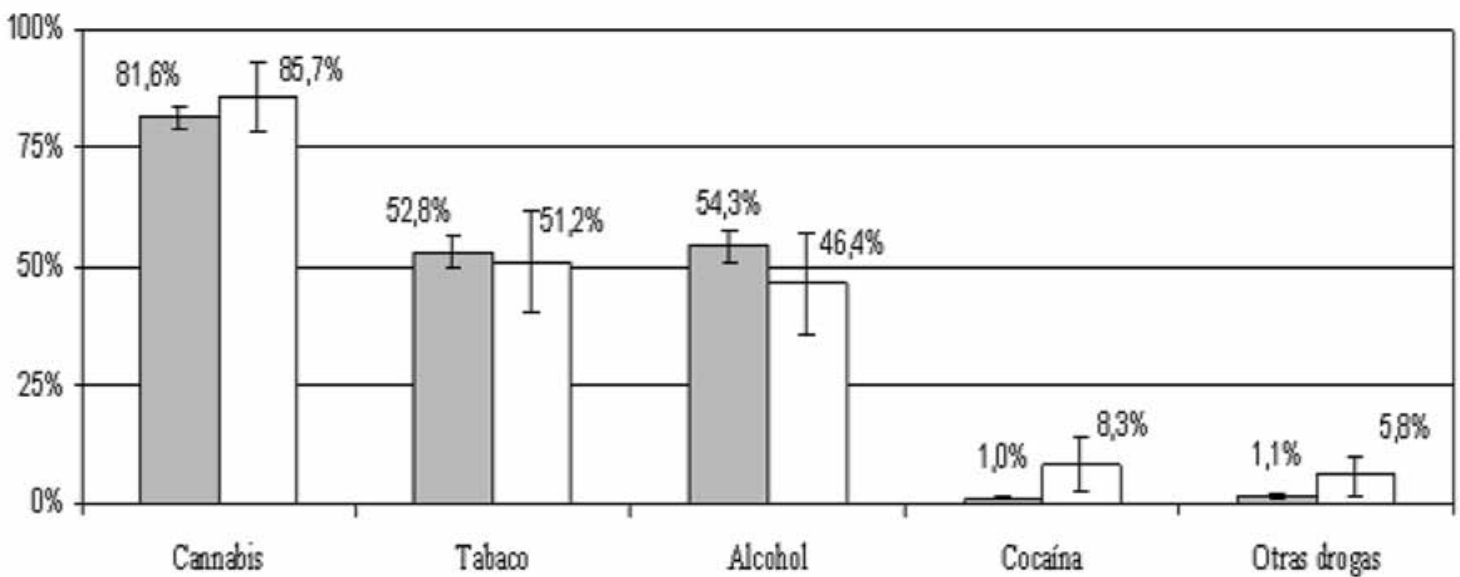

Programa de Medidas Alternativas (PMA) QPrograma de Información y Orientación(PIO) 
Tabla 2

Incidencia de casos atendidos en el programa de medidas alternativas (PMA) del Servicio de Orientación sobre Drogas, por edad. Barcelona, 2008-10

\begin{tabular}{|l|c|c|c|c|}
\hline \multicolumn{1}{|c|}{ Edad } & Número de casos & $\begin{array}{c}\text { Población de } \\
\text { Barcelona* }\end{array}$ & Tasa /1.000 personas-año & IC 95\% \\
\hline 14 años & 52 & 12.042 & 4,3 & $3,1-5,5$ \\
\hline 15 años & 149 & 12.300 & 12,1 & $10,2-14,1$ \\
\hline 16 años & 303 & 12.605 & 24 & $21,3-26,7$ \\
\hline 17 años & 367 & 12.974 & 28,3 & $25,4-31,2$ \\
\hline Total 14-17 años & 871 & 49.921 & 17,4 & $16,3-18,6$ \\
\hline
\end{tabular}

36 casos atendidos cumplieron los 18 años antes de iniciar el PMA.

(*) Departamento de Estadística del Ayuntamiento de Barcelona.

Tabla 3

Resultado de la intervención en las personas atendidas en el Servicio de Orientación sobre Drogas según programa. Barcelona, 2008-10

\begin{tabular}{|l|c|c|c|c|c|c|c|}
\hline & $\begin{array}{c}\text { Programa de } \\
\text { medidas } \\
\text { alternativas } \\
\text { (PMA) } \\
\mathrm{n}\end{array}$ & $\%$ & $\mathrm{IC} 95 \%$ & $\begin{array}{c}\text { Programa de } \\
\text { información y } \\
\text { orientación (PIO) } \\
\mathrm{n}\end{array}$ & $\%$ & IC95\% & $\mathrm{p}$ \\
\hline $\begin{array}{l}\text { Derivación a otros recursos tras } \\
\text { cribado inicial }\end{array}$ & 54 & 6,00 & $4,4-7,5$ & 39 & 37,90 & $28,5-47,2$ & $<0,0001$ \\
\hline $\begin{array}{l}\text { Derivación a Centro de Atención } \\
\text { y Seguimiento de la red de } \\
\text { drogodependencias }\end{array}$ & 18 & 2,00 & $-1,4-5,4$ & 29 & 28,20 & $19,5-36,8$ & \\
\hline $\begin{array}{l}\text { Derivación a Centro de Salud } \\
\text { Mental Infanto-juvenil }\end{array}$ & 36 & 4,00 & $-3,1-11,1$ & 10 & 9,70 & $4,0-25,4$ & \\
\hline Finalizan programa previsto & 840 & 92,60 & $90,9-94,3$ & 95 & 92,20 & $87,1-97,4$ & NS \\
\hline
\end{tabular}

otra parte, la proporción de adolescentes con un consumo de cannabis de bajo o moderado riesgo (CAST $=2-3$ ) fue de $22,7 \%$ en el PMA y del 13,2\% en el PIO.
La figura 1 presenta las sustancias que los participantes dijeron haber consumido en los 30 días anteriores al inicio del programa. Además del cannabis, destacó el alcohol como la sustancia más consumida con 523 
sujetos, 54,3\% en PMA y 46,4\%) en PIO, así como la presencia del tabaco en 514 $52,8 \%$ en PMA y $51,2 \%$ en PIO. El consumo de cocaína fue estadísticamente significativamente superior en el PIO, con un $8,3 \%$ frente al $1 \%$ en el PMA. En cuanto al consumo de otras drogas ilegales, había diferencias estadísticamente significativas, siendo superior el consumo en el PIO con un 5,8\% y $1,1 \%$ en el PMA.

La distribución por edad de los usuarios del PMA se presenta en la tabla 2. Como puede verse, a lo largo de la adolescencia se incrementó la probabilidad de entrar en el programa, aunque a los 1617 años pareció estabilizarse alrededor de 25 por mil adolescentes y año. La frecuencia de sujetos que acudieron al PMA fue de 17 adolescentes por mil personas de 14-17 años y año, y si se excluyeran los de 14 años esta tasa sería de 22,6 por mil. En la tabla 3 podemos observar los productos del SOD. El cribado facilitó que una proporción de personas fueran derivadas a un recurso especializado de salud mental o drogas, 39 (38\%) entre los usuarios del PIO y $54(6 \%)$ en el PMA $(p<0,0001)$. Las derivaciones a un centro de atención a las drogodependencias con programa específico para adolescentes fueron $29(28 \%)$ en los atendidos en el PIO y $18(2 \%)$ en el PMA ( $p<0,0001) .10$ $(9,7 \%)$ sujetos del PIO y $36(4 \%)$ del PMA $(p>0,05)$ fueron derivados a un centro de salud mental infanto-juvenil (CSMIJ). 935 adolescentes sin indicios de patología que entraron en el programa de intervención breve lo finalizaron, sin diferencias estadísticamente significativas entre PMA y PIO.

\section{DISCUSIÓN}

La puesta en marcha del PMA ha activado un circuito que comporta un flujo importante de menores al SOD, convirtiéndolo en la principal puerta de entrada al servicio. En general, los adolescentes atendidos en este programa hacen un consumo de cannabis (y en muchos casos de alcohol) de bajo o moderado riesgo, y finalizan la intervención propuesta. Son pocos los menores en el PMA que precisan de atención especializada por presentar criterios de abuso o dependencia a alguna sustancia psicoactiva $u$ otro trastorno psiquiátrico. Contrariamente, muchos de los usuarios del PIO acuden con problemas asociados al consumo, tanto de cannabis como de alcohol, más asentados y que inciden de una manera importante en sus vidas: la proporción que precisa ser derivada a un recurso especializado de salud mental o adicciones es de más de un tercio en este grupo, en el que por los criterios de entrada al programa, la edad media es más alta.

Además del valor de la intervención preventiva indicada sobre adolescentes cuyo consumo les ha llevado a entrar en una vía de sanción administrativa, el SOD facilita la detección precoz de consumos problemáticos de sustancias o de un trastorno mental incipiente y su derivación adecuada. Una de sus principales fortalezas reside en la capacidad de cribar y derivar a servicios especializados a consumidores que de otro modo probablemente no hubieran llegado a ellos hasta etapas más tardías, facilitando el tratamiento adecuado.

Como es natural, si se compara el perfil de los usuarios del SOD con los datos poblacionales de la encuesta ESTUDES de ámbito estatal, la proporción de sujetos con antecedentes de haber consumido cannabis y tabaco es mayor, especialmente la relativa al consumo de cannabis, que es cuatro veces superior en usuarios del SOD que en la población estudiantil de 14-18 años ${ }^{3}$. En esta misma encuesta se indica la estrecha asociación que hay entre los consumidores de cannabis y el consumo de alcohol y tabaco. La escala CAST permite valorar el consumo problemático de cannabis, entendido como 
aquel que genera problemas en el propio consumidor o en su entorno (de salud física, psíquica, problemas sociales o conductas de riesgo que pueden poner en peligro la salud del usuario) $)^{21}$ : Se observa que la prevalencia de consumidores de alto riesgo entre los usuarios del SOD es superior a la descrita en la población escolarizada de este grupo de edad en la ciudad en el estudio FRESC (5,3\% en las chicas y 7,3\% en los chicos de segundo de bachillerato o ciclos formativos de grado medio en 2008$)^{2}$, y por supuesto muy superior al 2\% del informe ESPAD de ámbito europeo ${ }^{27}$.

Los indicadores de frecuencia relativa de entrada en el PMA en relación a la población de la misma edad son relevantes. La probabilidad de entrar en el programa para la población de 14 a 17 años es del 17,4 por mil adolescentes de esta edad. Esto indica que la probabilidad de que un menor haya acudido al servicio durante su adolescencia para evitar sanciones por consumo de cannabis en la vía pública está próxima al $2 \%$. Esta cifra refleja la elevada prevalencia del consumo en la vía pública, más si tenemos en cuenta que sólo alrededor del 50\% de los jóvenes multados se acogen al PMA. Es sin duda la punta del iceberg, aunque como ya hemos dicho, según estudios previos, 6 de cada 10 jóvenes se inicia en el consumo en la vía publica ${ }^{18}$. Parece oportuno destacar el gran predominio de los varones y que la población con nacionalidad distinta a la española es algo menor que en la población de esta franja de edad en la ciudad (que es del 17,8\% para la franja de 15-19 años ${ }^{26}$ ).

Al responder el PMA a la aplicación del protocolo de colaboración entre las policías autonómica y local y la Agència de Salut Pública de Barcelona, que combina esta intervención de prevención indicada con las acciones en la vía pública de los cuerpos policiales, creemos que tiene un importante componente de prevención que va más allá de los usuarios individuales del PMA. La actuación policial en los espacios públicos podría producir un cambio del entorno y generar mensajes sociales educativos en contra de la banalización del consumo. Está demostrado que en nuestro medio la vía pública es el espacio de inicio para la mayoría de adolescentes ${ }^{28}$.

Entre las limitaciones y puntos débiles de este estudio cabe mencionar la posible existencia de sesgo de mala clasificación si hubiera falta de veracidad en algunas respuestas. Sin embargo, distintos estudios han demostrado una elevada fiabilidad en la información autorreferida con respecto al consumo de drogas ${ }^{29}$. También es una limitación no poder conocer las características de los adolescentes que no se acogen al programa PMA tras ser sancionados, por motivos éticos y de confidencialidad: la comparación con las características de los que sí siguen el programa sería de interés. Finalmente, la baja presencia de usuarias nos impide profundizar en el análisis por sexo.

Hemos utilizado el cuestionario AUDITC como instrumento de cribado, aunque su validación se realizó con población adulta. En España se utilizan habitualmente como puntos de corte de consumo de riesgo valores de 5 o más para varones y de 4 o más para mujeres, valores validados para la población adulta ${ }^{22}$. En el Manual del AUDIT se explicita que los puntos de corte utilizados han de tener en cuenta el contexto $^{30}$, por lo que al ser los adolescentes una población especialmente vulnerable ${ }^{31}$, optamos por utilizar con ellos el valor de corte definido para mujeres, opción sugerida también por una de las expertas en el instrumento que realizó estudios sobre su validez en medios clínicos en España (Rodríguez-Martos A, comunicación personal) ${ }^{32}$. Pensamos que sería deseable estudiar mejor las propiedades del AUDIT en esta franja de edad.

La tendencia a la evaluación de las intervenciones es creciente en promoción de la salud $^{33}$. El valor de una intervención breve extendida como la utilizada en estos progra- 
mas se ha demostrado en distintos trabajos $^{8,34,35}$, con efectos mantenidos incluso a medio plazo $^{36}$. Sin embargo, también hay algún estudio que proyecta dudas sobre su efectividad ${ }^{37}$, y además se ha usado sobre todo para abordar problemas relacionados con el alcohol. Por ello, aunque ya se ha invocado la necesidad de ofrecer intervenciones de esta naturaleza, hay que documentar su eficacia y efectividad durante su incipiente implantación en nuestro medio antes de proceder a su generalización en un contexto de escasos recursos ${ }^{7}$.

\section{AGRADECIMIENTOS}

Este trabajo no hubiera sido posible sin la dedicación de los profesionales del Servicio de Orientación sobre Drogas, ni sin la labor de campo de los profesionales del Cuerpo de Mossos d'Esquadra y de la Guardia Urbana de Barcelona y su colaboración en el Programa de Medidas Alternativas para menores sancionados por consumo y/o tenencia de drogas ilegales en la vía pública.

\section{BIBLIOGRAFÍA}

1 . DiClemente R, Santelli JS, Crosby RA (Eds). Adolescent health: understanding and preventing risk behaviors. San Francisco, CA, US: Jossey-Bass; 2009.

2 . Nebot M (Coord). Factors de risc en estudiants de secundària de Barcelona. Resultats principals de l'informe FRESC 2008. Barcelona: Agència de Salut Pública; 2010. Disponible en: http://www.aspb.cat/quefem/documents_enquestes.htm

3. Barrio G, Llorens N. El cielo revuelto del cannabis: ¿hay nueces para tanto ruido? Gac Sanit. 2008; 22: 297 299.

4. Plan Nacional sobre Drogas. Encuesta estatal sobre uso de drogas en estudiantes de secundaria (ESTUDES). Madrid: Ministerio de Sanidad y Políticas Sociales; 2009. Disponible en: http:/www.pnsd.msc.es/Categoria2/observa/pdf/Estudes2008.pdf.

5 . Gordon RS. An operational classification of disease prevention. Public Health Rep. 1983; 98: 107-109.

6. Burkhart G. Environmental drug prevention in the EU. Why is it so unpopular? Adicciones 2011; 23: 87-100.
7 . Toumbourou JW, Stockwell T, Neighbors C, Marlatt GA, Sturge J, Rhem J. Interventions to reduce harm associated with adolescent substance use. Lancet 2007; 369: 1391-1401.

8. Tait RJ, Hulse GK. A systematic review of the effectiveness of brief interventions with substance using adolescents by type of drug. University of York. Centre of Reviews and Dissemination (CRD). Database of Abstracts of Review of Effects (DARE). Disponible en: www.crd.york.ac.uk/CRDWeb/ShowRecord.asp?View= Full\&ID=12003008594.

9 . European Monitoring Centre for Drugs and Drug Addiction. Preventing later substance use disorders in at risk children and adolescents. A review of the theory and evidence base of indicated prevention. Luxembourg: Office for Official Publications of the European Communities; 2009 . Disponible en: http://www.emcdda.europa.eu/attachements.cfm/att_69 429_EN_EMCDDA-TB-indicated_prevention.pdf.

10 . Grenard JL, Ames SL, Pentz MA, Sussman S. Motivational interviewing with adolescents and young adults for drug-related problems. Int J Adolesc Med Health. 2006; 18: 53-67.

11. Vasilaki EI, Hosier SG, Cox WM. The efficacy of motivational interviewing as a brief interventions for excessive drinking: a meta-analytic review. Alcohol Alcohol. 2006; 41:328-335.

12. Monti PM, Barnet NP, Colby SM, Gwaltney CJ, Spirito A, Rohsenow DJ, et al. Motivational interviewing versus feedback only in emergency care for young adult problem drinking. Addiction. 2007: 102: 1234-1243.

13. Spirito A, Sindelar-Manning H, Colby SM, Barnett NP, Lewnder W, Rohsenow DJ, Monti PM. Individual and family motivational interventions for alcohol-positive adolescents treated in an emergency department: results of a randomized clinical trial. Arch Pediatr Adolesc Med. 2011; 165: 269-274

14. D’Amico EJ, Miles JN, Stern SA, Meredith LS. Brief motivational interviewing for teens at risk of substance use consequences: a randomized pilot study in a primary care clinic. J Subst Abuse Treat. 2008; 35:53-61.

15. Stein LA, Colby SM, Barnett NP, Monti PM, Golembeske C, Lebeau-Craven R. Effects of motivational interviewing for incarcerated adolescents on driving under the influence after release. Am J Addict. 2006; 15 Supl 1: 50-57.

16. Grenard JL, Ames SL, Wiers RW, Tus C, Stacy AW, Sussman S. Brief intervention for substance use among at-risk adolescents: A pilot study. J Adoles Health. 2007; 40:188-191. 
17. Suárez-Vázquez R. Menores consumidores. Factores de riesgo. Adicciones. 2006; 17 Supl 1: 54.

18 . National Institute for Health and Clinical Excellence. Alcohol-use disorders: preventing the development of hazardous and harmful drinking. London: NICE Public Health Guidance; 2010. Disponible en http://www.nice.org.uk/nicemedia/live/13001/48984/ 48984.pdf.

19. Agència de Salut Pública de Barcelona. La salut a Barcelona 2008. Barcelona: Agència de Salut Pública de Barcelona; 2009.

20. Morales E, Ariza C, Nebot M, Pérez A, Sánchez F. Consumo de cannabis en los estudiantes de secundaria de Barcelona: inicio en el consumo, efectos experimentados y expectativas. Gac Sanit. 2008; 22:321-329.

21. Room R, Babor TF, Rehm J. Alcohol and public health. Lancet 2005; 365:519-530.

22. Gual A, Segura L, Contel M, Heather N, Colom J. Audit-3 and Audit-4: effectiveness of two short forms of the alcohol use disorders identification test. Alcohol Alcohol. 2002;37:591-596

23. Klempova D, Sánchez A, Vicente J, Barrio G, Domingo A, Suelves JM, Llorens N, Prieto L, Brime B, Ramírez V. Consumo problemático de cannabis en adolescentes españoles de 14-18 años. Validación de escalas. Madrid: Delegación del Gobierno para el Plan Nacional sobre Drogas; 2009. Disponible en http://www.pnsd.msc.es/Categoria2/publica/pdf/ConsProblematico_cannabis.pdf

24. Knight JR, Goodman E, Pulerwitz T, DuRant RH. Reliability of the Problem Oriented Screening Instrument for Teenagers (POSIT) in adolescent medical practice. J Adolesc Health. 2001; 29:125-130.

25 . Prochaska JO, DiClemente CC. Towards a comprehensive model of change. In Prochaska JO, DiClemente CC (eds) The transtheoretical approach: crossing the traditional boundaries of therapy. Homewood (IL): DowJones; 1984.

26. Departament d'Estadística. Estadístiques de població i llars. Barcelona: Ajuntament de Barcelona;2011. Disponible en: http://www.bcn.cat/estadistica/catala/dades/ tpob/index.htm.

27 . Observatorio Europeo de las Drogas y Toxicomanías. Resumen informe ESPAD 2007. Consumo de sustancias entre escolares de 35 países europeos. Luxembourg: Office for Official Publications of the European Communities, 2009. Disponible en: http://www.emcdda.europa.eu/attachements.cfm/att_77168_ES_ESPAD_summary_ES.pdf
28. Morales E, Ariza C, Nebot M, Pérez A, Sánchez F. Consumo de cannabis en los estudiantes de secundaria de Barcelona: inicio en el consumo, efectos experimentados y expectativas. Gac Sanit. 2008; 22: 321-329.

29. Jungerman FS, Andreoni S, Laranjeira R. Short term impact of same intensity but different duration interventions for cannabis users. Drug Alcohol Depend. 2007; 90:120-127.

30. Babor TF, Higgins-Biddle JC, Saunders JB, Monteiro MG. AUDIT. Cuestionario de Identificación de los Transtornos debidos al Consumo de Alcohol. Pautas para su utilización en Atención Primaria. Ginebra: World Health Organization; 2001. Disponible en http://www.who.int/substance_abuse/activities/en/AUDITmanualSpanish.pdf

31. Guerri C, Pascual M. Mechanisms involved in the neurotoxic, cognitive, and neurobehavioral effects of alcohol consumption during adolescence. Alcohol. 2010; 44:15-26.

32. Rodríguez-Martos A., Santamariña E. Does the short form of the Alcohol Use Disorders Identification Test (AUDIT-C) work at a trauma emergency department? Subst Use Misuse. 2007;46: 923-932.

33. Hernán García M, Ramos Montserrat M, Fernández Jauría A. Revisión de los trabajos publicados sobre promoción de la salud en jóvenes españoles. Rev Esp Salud Pública. 2001; 75: 491-504.

34. Martin G, Copeland J. The adolescent cannabis check-up: randomized trial of a brief intervention for young cannabis users. J.Subst.Abuse Treat. 2008;34:407414.

35 . McCambridge J, Strang J. The efficacy of singlesession motivational interviewing in reducing drug consumption and perceptions of drug-related risk and harm among young people: results from a multi-site cluster randomized trial. Addiction. 2004;99:39-52.

36. Stephens RS, Roffman RA, Fearer SA, Williams C, Burke RS. The Marijuana Check-up: promoting change in ambivalent marijuana users. Addiction. 2007;102:947-957.

37. White HR, Morgan TJ, Pugh LA, Celinska K, Labouvie EW, Pandina RJ. Evaluating two brief substance-use interventions for mandated college students. J Stud Alcohol. 2006;67:309-317. 